\title{
Power Flow Study of Low-Voltage DC Micro-Grid and Control of Energy Storage System in the Grid
}

\author{
Dong-Eok Kim ${ }^{\dagger}$
}

\begin{abstract}
DC distribution has several differences compared to AC distribution. DC distribution has a higher efficiency than $\mathrm{AC}$ distribution when distributing electricity at the same voltage level. Accordingly, power can be transferred further with low-voltage DC. In addition, power flow in a DC grid system is produced by only a voltage difference in magnitude. Owing to these differences, operation of a DC grid system significantly differs from that of an AC system. In this paper, the power flow problem in a bipolar-type DC grid with unbalanced load conditions is organized and solved. Control strategy of energy storage system on a slow time scale with power references obtained by solving an optimization problem regarding the DC grid is then proposed. The proposed strategy is verified with computer simulations.
\end{abstract}

Keywords: DC micro-grid, Bipolar-type DC distribution, DC power flow, Energy storage system

\section{Introduction}

Nowadays, most electrical equipment, such as servo motor drives, renewable generation systems, and information technology devices, apply a power conversion process with power electronics to enhance energy efficiency and performance. This power conversion typically uses a pulse width modulation (PWM) technique, which requires a DC source. However, because electricity is distributed in the form of $\mathrm{AC}$, an additional conversion process of $\mathrm{AC}$ to $\mathrm{DC}$ is required for the DC source, which results in reduced energy efficiency. For these reasons, DC distribution is receiving considerable attention. Many studies and attempts have been made to distribute electricity in DC form [1-3]. DC distribution is predominantly required for internet data centers (IDCs). IDCs require multiple uninterruptible power supplies (UPSs), which require redundant AC to DC conversion processes [4]. These processes can be avoided if DC distribution is used. Furthermore, DC distribution networks have been configured in commercial buildings to enhance energy efficiency with photovoltaic (PV) systems [5].

In addition, because the DC system has no inductance components in a steady state, electricity can be further transferred by DC distribution at a low-voltage level compared to that by AC. Some studies showed that transferring electricity in DC low-voltage level is more efficient than transferring electricity at an AC high-voltage level to some distance [6], as well as better power quality $[7,8]$. In developing countries, the DC micro-grid with a small PV system has been applied for rural area electrification [9]. In [10], the DC micro-grid with

\footnotetext{
Corresponding Author: KEPCO Research Institute, Korea Electric Power Corporation, Daejeon, Korea. (cscotop@hanmail.net) Received: June 8, 2016; Accepted: December 28, 2016
}

renewable resources was applied on a small scale on an island. In [11], to prevent a total black-out by a natural disaster, a low-voltage DC micro-grid in a complex building area was constructed, and it proved to be successful. Furthermore, when applying renewable generation systems to a DC grid, synchronization no longer needs to be considered [12].

Although DC technology is common and matured, designing and operating a DC micro-grid is a different matter. There are things to be studied and verified, such as a grounding problem in the grid. In addition, a DC grid system differs from an AC grid system in many aspects. Especially, power flow in DC system is made only by voltage difference in magnitude. Further, in the bipolar type of DC distribution, loads can be connected to either positive or negative side [13] so that unbalanced power flow occurs. This unbalanced problem might be severe if the distribution lines are extended over a large area, which results in unbalanced voltage in the grid, and thus it must be taken into accounts when analyzing and operating a bipolar-type DC micro-grid. However, most studies for DC micro-grid are concerned with unipolar types and they are not focused on the unbalanced conditions [14-16]

In a micro-grid, renewable resources are essential as well as energy storages. When renewable resources are applied in a micro-grid system, single or multiple energy storage systems (ESSs) typically are installed in the grid and they support the operation of the grid by properly charging or discharging power. In addition to that, ESS can work to eliminate unbfigced power flow in the bipolar DC micro-grid.

In this paper, we first introduce how to organize and solve a power flow problem for a bipolar-type DC microgrid system. By using it, we can study the unbalanced phenomenon caused by unbalanced load demand or power 

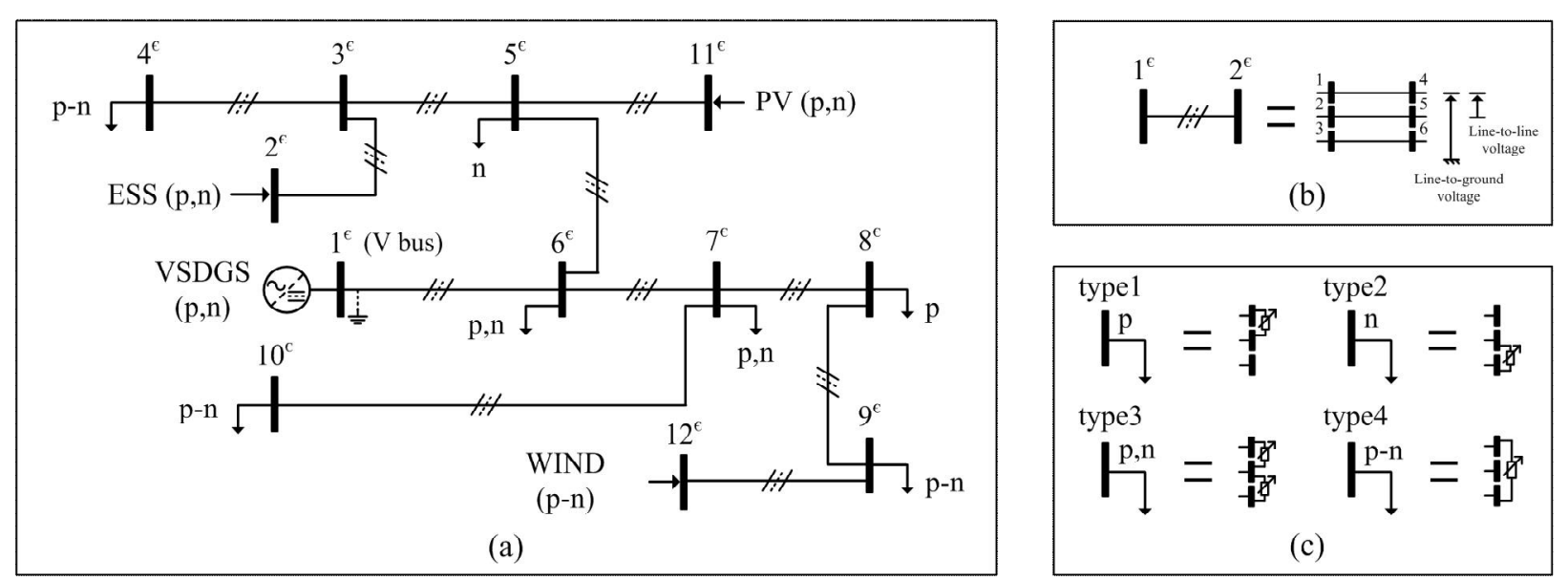

Fig. 1. (a) Bipolar-type DC micro-grid represented in a single-line diagram; (b) Line between two buses in a single-line diagram and its representation in a three-line diagram; (c) Four types of loads represented in single-line and threeline diagrams

generation in the grid. Second, to resolve the unbalance problem, we present the control strategy of ESS, by which it can support voltages as well as proper power flow in the grid. The strategy is concerned with quasi-static control action on a slow time scale, which is similar with secondary control in AC power system to maintain frequency of a wide area within desired bounds [17].

\section{DC Micro-Grid}

Although the DC micro-grid configuration and operation can be achieved in many ways, proper voltage control is important because power in a DC grid flows only by voltage differences in magnitude. And, this work focuses on ESS operation on a slow time scale by an energy management system (EMS) to support line (line-to-line) voltages as well as proper power flow in a DC micro-grid. For this, we first need to configure power flow problems and solve them to determine the operating condition of the DC micro-grid.

\subsection{Bipolar-type DC grid}

Fig. 1(a) shows a bipolar-type DC micro-grid represented in a single-line diagram. Each bus numbered with the superscript ' $\epsilon$ ' includes three buses - positive, neutral, and negative buses - as shown in Fig. 1(b). Bus numbers in the single-line and three-line diagrams have the following relations:

$$
\left\{i \mid i \in N, 3\left(i^{\epsilon}-1\right)+1 \leq i \leq 3 i^{\epsilon}\right\}
$$

where $i^{\epsilon} \in N$ and $1 \leq i^{\epsilon} \leq n$. The grid system includes a variable-speed diesel-engine generation system (VSDGS), distributed renewable generation systems (DRGSs) using PV and wind, ESS, and loads. Connection to the grid can be made by four types, which are shown in Fig. 1(c). Generation systems with the capability of power redistribution for voltage balance in the grid must be connected by the $(\mathrm{p}, \mathrm{n})$ type, while loads are connected by all types.

For this work, several assumptions are made: 1) EMS can have information of voltages, currents, and powers of buses and can use them for the grid operation. EMS transfers control references to controllable systems, and the systems can correctly follow the references. 2) VSDGS is connected to the grid by $\mathrm{AC} / \mathrm{DC}$ converters so it can run efficiently over all operation ranges. This VSDGS system also has the capability of controlling the line voltage of bus $1^{\epsilon}$ with the converters, where the voltage reference is given by EMS. In addition, a controllable dumping load is connected to VSDGS. This implies that, when power is surplus in the grid, the system can absorb the power. 3) DRGSs are usually controlled with maximum power point tracking (MPPT) method; however, they can reduce its output power by an EMS order when the grid has surplus power. 4) Normal loads cannot be intentionally shed or controlled. However, a public thermal load, such as a combined heat and power (CHP) system, is controllable to some extent if any exists.

\subsection{Power flow problem for bipolar-type DC grid}

In Fig. 1, the bus type is categorized into two bus types: $\mathrm{V}$ and $\mathrm{P}$ buses. The $\mathrm{V}$ bus is a reference bus numbered $1^{\epsilon}$ (or 1, 2, and 3) so that all bus voltages are calculated based on this particular bus voltage. The others are $\mathrm{P}$ buses into which generated powers are injected. Power demands by loads are represented as negative power injections. Loads are connected between buses and require constant powers. Loads are categorized into four types, as shown in Fig. 1(c).

When the line conductance matrix of the DC network represented in a single-line diagram is given $G_{A}^{\epsilon}$, that of the three-line diagram is derived by using the Kronecker 
product:

$$
\boldsymbol{G}_{A}=\boldsymbol{G}_{A}^{\epsilon} \otimes \boldsymbol{I}_{3 \times 3}
$$

where $\boldsymbol{I}_{3 \times 3}$ is a $3 \times 3$ identity matrix. Because loads require constant powers, they are assumed resistances that vary depending on line voltage changes. In addition, DRGSs are considered negative loads. Then, the conductance matrix can be decomposed into those of DC-network lines and loads

$$
\boldsymbol{G}=\boldsymbol{G}_{A}+\boldsymbol{G}_{L}
$$

where $\boldsymbol{G}_{L}$ is the conductance matrix of the loads. To solve the power flow problem with the Newton-Raphson method, we must develop the respective power equations and Jacobean matrix [18]. First, current injected into $i^{\text {th }}$ bus is calculated as

$$
I_{i}=\sum_{k=1}^{3 n} G_{i k} V_{k}=\sum_{k=1}^{3 n} G_{A, i k} V_{k}+\sum_{k=1}^{3 n} G_{L, i k} V_{k}
$$

which is expressed in a matrix form, $\boldsymbol{I}=\boldsymbol{G} \boldsymbol{V}$. Then, power injected into $i^{\text {th }}$ bus is

$$
P_{i}=V_{i} I_{i}
$$

Also, powers consumed by the loads are calculated as

$$
P_{L, p}=\left(V_{r}-V_{s}\right)^{2} g_{L, p}=-\left(V_{r}-V_{s}\right)^{2} G_{L, r s}
$$

where $P_{L, p}$ is the power demanded by the $p^{\text {th }}$ load connected between $r$ and $s$ buses. $g_{L, p}$ is the conductance of the $p^{\text {th }}$ load. Using (5) and (6), the following can be obtained



$$
\boldsymbol{x}^{v+1}=\boldsymbol{x}^{v}+\boldsymbol{J}\left(\boldsymbol{x}^{v}\right)^{-1} \boldsymbol{f}\left(\boldsymbol{x}^{v}\right)
$$

where

$$
\begin{aligned}
& \boldsymbol{J}(\boldsymbol{x})=\left(\begin{array}{ll}
\boldsymbol{J}_{a} & \boldsymbol{J}_{b} \\
\boldsymbol{J}_{c} & \boldsymbol{J}_{d}
\end{array}\right), \boldsymbol{f}(\boldsymbol{x})=\left(\begin{array}{c}
\Delta \boldsymbol{P}_{(4-\cdot)} \\
\Delta \boldsymbol{P}_{L}
\end{array}\right), \boldsymbol{x}=\left(\begin{array}{c}
\boldsymbol{V}_{(4-\cdot)} \\
\boldsymbol{g}_{L}
\end{array}\right) \\
& J_{a, i j}=\frac{\partial P_{i}}{\partial V_{j}}, J_{b, i q}=\frac{\partial P_{i}}{\partial g_{L, q}}, J_{c, p j}=\frac{\partial P_{L, p}}{\partial V_{j}}, J_{d, p q}=\frac{\partial P_{L, p}}{\partial g_{L, q}}, \\
& \Delta P_{i}=P_{i}-P_{i}^{*}, \Delta P_{L, p}=P_{L, p}-P_{L, p}^{*} .
\end{aligned}
$$

$\boldsymbol{J}$ is a Jacobean matrix, and superscript $v$ indicates the iteration number. It should be noted that the $\mathrm{V}$ bus voltage is fixed at a given value and not included in $\boldsymbol{x}$. In addition, it is important to note that, because all loads, including negative loads, are represented conductance, the power injected into buses is zero over the iteration process $\left(P_{i}^{*}=0\right)$. Once the solution is obtained, the power injected can be calculated using the bus voltages and line conductance.

\section{ESS Operation in the DC Grid}

In this work, a VSDGS is considered as a reference unit that is controlled to maintain its terminal ( $\mathrm{V}$ bus) line voltage to a given value (1.0 pu). Once DRGSs are built, an optimal solution is to enable them to generate power as much as possible, and that can be achieved by a simple principle of : while DRGSs generate their maximum powers, ESS charges or discharges to maintain the line voltages of all buses as close as that of the $\mathrm{V}$ bus in the grid.

To do that, we solve an optimization problem to obtain the proper power reference and ESS is controlled to produce the same power as the reference. However, this
(A1) For Mode 1

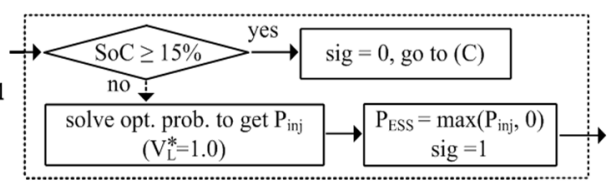

(A2)
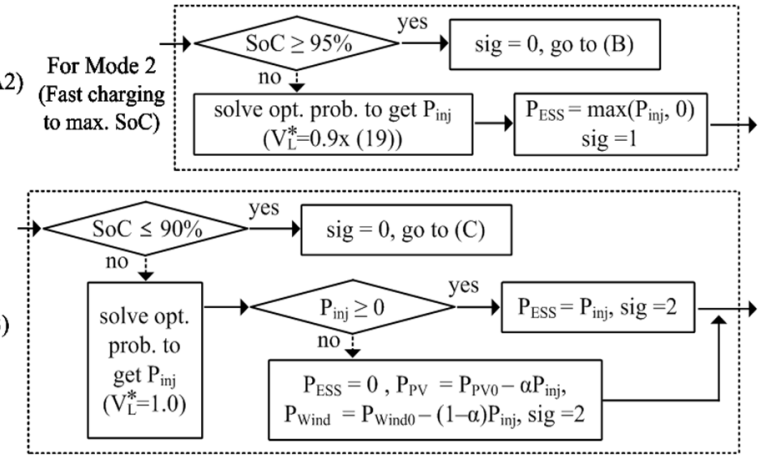

(C) $\rightarrow$ Solve opt. prob. to get $\mathrm{P}_{\text {inj }}\left(\mathrm{V}_{\mathrm{L}}^{*}=1.0\right) \longrightarrow \mathrm{P}_{\mathrm{ESS}}=\mathrm{P}_{\mathrm{inj}}$, sig $=0$

Fig. 2. Flow chart of the ESS operation (generating power references continuously every minute unless interrupted by an external signal) 
power reference might not be fulfilled if the ESS's stateof-charge $(\mathrm{SoC})$ is lower than its low limit or higher than its upper limit. If $\mathrm{SoC}$ is lower than its low limit, ESS cannot discharge; it only charges where the energy for the charging comes from the VSDGS system and DRGSs. If $\mathrm{SoC}$ is higher than its upper limit, ESS can no longer charge, and it contributes voltage support by only discharging to the grid. In this case, if DRGSs generate too much power, a power surplus might occur in the grid. Then, 1) the generation from DGs should be reduced, 2) power consumption of loads such as CHP should be increased, or 3 ) the surplus power should be wasted by some resistors at VSDGS. Considering these operational conditions, a flow chart for the ESS operation is derived and shown in Fig. 2.

We first choose mode 1 or 2 . ESS is predominantly charged from DRGSs with mode 1, whereas ESS is rapidly charged by energy from both VSDGS and DRGSs with mode 2. This is because ESS is operated to make line voltages around the predetermined value of $0.9 x$ (recall the VSDGS line voltage of $1.0 \mathrm{pu}$ ). For instance, let us say that mode 1 is chosen and $\mathrm{SoC}$ is $10 \%$. Then, we transition to (A1). Since $\mathrm{SoC}$ is less than $15 \%$, we solve the optimization problem and obtain $P_{\text {inj. }}$. At this point, ESS cannot discharge; therefore, the ESS power reference, $P_{\mathrm{ESS}}$, should be less than 0 , even if the solution is larger than 0 . Then, the sig sign is set to 1 and then do the process again. If $\mathrm{SoC}$ is larger than $15 \%$, the sig sign is set to 0 and we go to $(\mathrm{C})$.

Aside from the logic explained above, the main part of the operation is to solve the optimization problem. The next two sub-sections explain the optimization problem to generate ESS power references.

\subsection{Constraints by Kirchhoff's law and constant power load}

This sub-section outlines the physical relations to be met in the optimization problem. First, the V bus voltage is fixed and expressed in per unit form:

$$
\boldsymbol{V}_{1^{\epsilon}}=\left(\begin{array}{l}
V_{1} \\
V_{2} \\
V_{3}
\end{array}\right)=\left(\begin{array}{c}
1 \\
0 \\
-1
\end{array}\right)
$$

Second, Kirchhoff's circuit law should be satisfied. From (4), the current equation is expressed in a matrix form

$$
\Delta \boldsymbol{I}\left(=\boldsymbol{I}-\boldsymbol{I}_{o}\right)=\boldsymbol{G}_{A} \Delta \boldsymbol{V}\left(=\boldsymbol{G}_{A}\left(\boldsymbol{V}-\boldsymbol{V}_{o}\right)\right)
$$

where $\Delta \boldsymbol{I}=\left(\begin{array}{lll}\Delta I_{1} & \cdots & \Delta I_{3 n}\end{array}\right)^{T}, \quad \Delta \boldsymbol{V}=\left(\begin{array}{lll}\Delta V_{1} & \cdots & \Delta V_{3 n}\end{array}\right)^{T}$, $\Delta I_{i}=I_{i}-I_{o, i}$ and $\Delta V_{i}=V_{i}-V_{o, i}$. Symbol $\Delta$ is a difference operator which represents the change of a variable from a given operating point. Subscript 'o' signifies the operating points, which are obtained by solving the
Table 1. Relations of load type and current

\begin{tabular}{c|c}
\hline No load & $I_{L, \cdot}^{+}=0, \quad I_{L, \cdot}^{-}=0$ \\
\hline Load type 1 (p) & $I_{L,}^{+} \neq 0, \quad I_{L,}^{-}=0$ \\
\hline Load type 2 (n) & $I_{L,}^{+}=0, \quad I_{L,}^{-} \neq 0$ \\
\hline Load type 3 (p,n) & $I_{L, \cdot}^{+} \neq 0, \quad I_{L,}^{-} \neq 0$ \\
\hline Load type 4 (p-n) & $I_{L, \cdot}^{+}=I_{L,}^{-} \neq 0$ \\
\hline
\end{tabular}

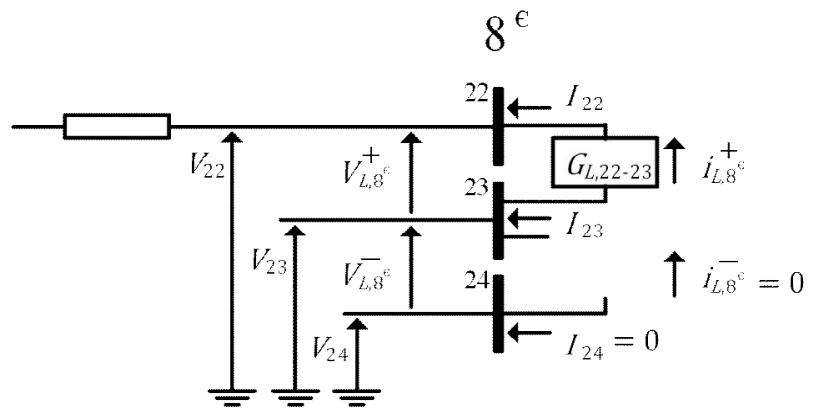

Fig. 3. Load at bus $8^{\epsilon}$ in Fig. 1 (load type 1 (p))

power flow problem. Here, (9) must be modified to be represented in terms of load currents. To this end, we must derive the relations between the current injected into a certain bus and load current as

$$
\begin{aligned}
& \left(\begin{array}{c}
I_{3(i-1)+1} \\
I_{3(i-1)+2} \\
I_{3 i}
\end{array}\right)=\left(\begin{array}{cc}
1 & 0 \\
-1 & 1 \\
0 & -1
\end{array}\right)\left(\begin{array}{c}
I_{L, i^{\epsilon}}^{+} \\
I_{L, i^{\epsilon}}^{-}
\end{array}\right), \\
& \left(\begin{array}{c}
I_{L, i^{\epsilon}}^{+} \\
I_{L, i^{\epsilon}}^{-}
\end{array}\right)=\left(\begin{array}{ccc}
1 & 0 & 0 \\
0 & 0 & -1
\end{array}\right)\left(\begin{array}{c}
I_{3(i-1)+1} \\
I_{3(i-1)+2} \\
I_{3 i}
\end{array}\right)
\end{aligned}
$$

where superscript '+' and ' - ' signify the positive side (between positive and neutral buses) and negative side (between neutral and negative buses), respectively. For instance, $I_{L, g^{\epsilon}}^{+}$is the current demanded by a load connected to the positive side of bus $8^{\epsilon}$ in single diagram (a load connected between bus 22 and 23). Regarding (10), the relations in Table 1 are satisfied depending on the type of loads.

And, the bus voltage and load voltage (line voltage) have the following relations:

$$
\left(\begin{array}{c}
V_{L,,^{\epsilon}}^{+} \\
V_{L, i^{\epsilon}}^{i}
\end{array}\right)=\left(\begin{array}{ccc}
1 & -1 & 0 \\
0 & 1 & -1
\end{array}\right)\left(\begin{array}{c}
V_{3(i-1)+1} \\
V_{3(i-1)+2} \\
V_{3 i}
\end{array}\right)
$$

where $V_{L, i^{\epsilon}}^{+}$and $V_{L, i^{\epsilon}}^{-}$represent the line voltages of $i^{\epsilon}$ bus's positive and negative sides, respectively. For a clearer understanding, Fig. 3 shows an example for (10) and (11) with a load connected at the positive side of bus $8 \epsilon$. Now, by modifying (9) using (10) and (11), we obtained a set of equality conditions for Kirchhoff's circuit law:

$$
\left(\begin{array}{ll}
-\boldsymbol{G}_{A} & \boldsymbol{K}_{I}
\end{array}\right)\left(\begin{array}{l}
\boldsymbol{V} \\
\boldsymbol{I}_{a}
\end{array}\right)=-\boldsymbol{G}_{A} \boldsymbol{V}_{o}+\boldsymbol{K}_{I} \boldsymbol{I}_{a o}(=\mathbf{0})
$$


where

$$
\begin{aligned}
& \boldsymbol{I}_{a}=\left(\begin{array}{c}
\boldsymbol{I}_{1^{\epsilon}} \\
\boldsymbol{I}_{L}
\end{array}\right): \boldsymbol{I}_{1^{\epsilon}}=\left(\begin{array}{c}
I_{1} \\
I_{2} \\
I_{3}
\end{array}\right), \boldsymbol{I}_{L}=\left(\begin{array}{c}
\boldsymbol{I}_{L, 2^{\epsilon}} \\
\vdots \\
\boldsymbol{I}_{L, n^{\epsilon}}
\end{array}\right), \boldsymbol{I}_{L, i^{\epsilon}}=\left(\begin{array}{c}
I_{L, i^{\epsilon}}^{+} \\
I_{L, i^{\epsilon}}^{-}
\end{array}\right), \\
& \boldsymbol{V}=\left(\begin{array}{c}
\boldsymbol{V}_{1^{\epsilon}} \\
\vdots \\
\boldsymbol{V}_{n^{\epsilon}}^{\epsilon}
\end{array}\right): \boldsymbol{V}_{i^{\epsilon}}=\left(\begin{array}{c}
V_{3(i-1)+1} \\
V_{3(i-1)+2} \\
V_{3 i}
\end{array}\right),
\end{aligned}
$$

and $\boldsymbol{K}_{I}$ is obtained by (10).

In addition, we need a set of equality conditions for constant power demand by loads or constant power generation by DRGSs. From that constant power means the change is zero, the following is derived in accordance with a small signal modelling technique,

$$
\left(\begin{array}{l}
\Delta P_{L, i^{\epsilon}}^{+}(=0) \\
\Delta P_{L, i^{\epsilon}}^{-}(=0)
\end{array}\right)=\left(\begin{array}{l}
I_{L o, i}^{+} \Delta_{L, i^{\epsilon}}^{+}+V_{L o, i}^{+} \Delta I_{L, i^{\epsilon}}^{+} \\
I_{L o, i}^{-} \Delta V_{L, i^{\epsilon}}^{-}+V_{L o, i}^{-} \Delta I_{L, i^{\epsilon}}^{-}
\end{array}\right)
$$

where $\Delta P_{L, i} \epsilon$ is the change of power demand by loads (or power generation by DRGSs) connected at $i^{\epsilon}$ bus. $\Delta V_{L, i^{\epsilon}}=V_{L, i^{\epsilon}}-V_{L o, i^{\epsilon}}$ and $\Delta I_{L, i^{\epsilon}}=I_{L, i^{\epsilon}}-I_{L o, i^{\epsilon}}$. Using (11) and (13), a constraint set regarding the constant power demand is derived:

$$
\left(\begin{array}{ll}
\boldsymbol{K}_{1} & \boldsymbol{K}_{2}
\end{array}\right)\left(\begin{array}{l}
\boldsymbol{V} \\
\boldsymbol{I}_{a}
\end{array}\right)=2 \boldsymbol{K}_{2} \boldsymbol{I}_{a o}
$$

Here, it needs to note that, as the powers of VSDGS and ESS are not constrained by the condition of (13), the corresponding elements of $\boldsymbol{K}_{1}$ and $\boldsymbol{K}_{2}$ in (14) are zeros.

\subsection{Optimization problem for ESS operation}

Single or multiple ESSs can support the voltage balance in a DC micro-grid by properly charging or discharging power from them. For that, we need to derive the power references of ESS by solving an optimization problem. In this section, we consider a case of single ESS.

The objective of the optimization problem is to maintain all line voltages as close to a given reference $\left(V_{L}^{*}\right)$ as possible, which can be achieved by minimizing $\left(\boldsymbol{V}_{L}-\right.$ $\left.\mathbf{1} V_{L}^{*}\right)^{T}\left(\boldsymbol{V}_{L}-\mathbf{1} V_{L}^{*}\right)$. Using (11), a quadratic cost function is expressed as

$$
\boldsymbol{x}^{T} \boldsymbol{H} \boldsymbol{x}-2\left(\mathbf{1} V_{L}^{*}\right)^{T} \boldsymbol{F} \boldsymbol{x}
$$

where $\boldsymbol{x}=\boldsymbol{V}, \boldsymbol{H}=\boldsymbol{F}^{T} \boldsymbol{F}$, and matrix $\boldsymbol{F}$ is obtained by the relation $\boldsymbol{V}_{L}=\boldsymbol{F} \boldsymbol{V}$ of (11).

The current magnitude of ESS should be smaller than a rated value. Assuming that ESS is connected to the $m^{\text {th }}$ bus,

$$
\left(\begin{array}{l}
I_{L, m^{\epsilon}}^{+} \\
I_{L, m^{\epsilon}}^{-}
\end{array}\right) \leq\left(\begin{array}{l}
I_{l i m} \\
I_{l i m}
\end{array}\right),\left(\begin{array}{cc}
-1 & 0 \\
0 & -1
\end{array}\right)\left(\begin{array}{l}
I_{L, m^{\epsilon}}^{+} \\
I_{L, m^{\epsilon}}^{-}
\end{array}\right) \leq\left(\begin{array}{l}
I_{l i m} \\
I_{l i m}
\end{array}\right)
$$

And, to avoid power flow into a generator system connected to the V bus (except during the transient state),

$$
\left(\begin{array}{cc}
-1 & 0 \\
0 & 1
\end{array}\right)\left(\begin{array}{l}
I_{1} \\
I_{3}
\end{array}\right) \leq\left(\begin{array}{l}
0 \\
0
\end{array}\right)
$$

In some cases that the power of DRGSs is larger than the power to be consumed by loads - but ESS is fully charged - surplus power must be wasted by some resistors with VSDGS. Thus, SoC is checked before solving the optimization problem if it is higher than the upper limit. If so, (17) is removed from the constraint set. By applying the cost function (14), equality conditions $(8,12,14)$, and inequality conditions $(16,17)$, the optimization problem is organized as

$$
\begin{array}{ll}
\text { Minimize } & \boldsymbol{x}^{T} \boldsymbol{H} \boldsymbol{x}-2\left(\mathbf{1} V_{L}^{*}\right)^{T} \boldsymbol{F} \boldsymbol{x} \\
\text { Subject to } & \boldsymbol{A}_{e q} \boldsymbol{z}=\boldsymbol{b}_{e q}, \boldsymbol{A}_{\text {ineq }} \boldsymbol{z} \leq \boldsymbol{b}_{\text {ineq }}
\end{array}
$$

where $\boldsymbol{z}=\left(\begin{array}{c}\boldsymbol{x}(=\boldsymbol{V}) \\ \boldsymbol{I}_{a}\end{array}\right), \boldsymbol{A}_{e q} \boldsymbol{z}=\boldsymbol{b}_{\text {eq }}$ and $\boldsymbol{A}_{\text {ine } q} \mathbf{z} \leq \boldsymbol{b}_{\text {ineq }}$ are the equality and inequality constraints in matrix form, respectively. The problem is solved using a tool such as CVX, which is the name of a MATLAB software for disciplined convex programming [19], or numerically solved by the Karush-Kuhn-Tucker (KKT) condition.

In addition, reference $V_{L}^{*}$ in $(15)$ can be otherwise set depending on the charging mode, as shown in Fig. 2. Normally, $V_{L}^{*}$ is set to be the same as the $\mathrm{V}$ bus line voltage $\left(V_{L}^{*}=1.0\right)$ so it can minimize the power from VSDGS. However, when we need to rapidly charge ESS for certain purposes (mode 2 in Fig. 2), this can be accomplished by setting $V_{L}^{*}$ to be less than the $\mathrm{V}$ bus line voltage. To calculate a proper reference for it, we must derive a reduced network that has only the $\mathrm{V}$ bus and $m^{\text {th }}$ bus that ESS is connected. We can assume the current remains constant during a control period $\left(\Delta I_{i}=0\right)$, except for the $\mathrm{V}$ bus and $m^{\text {th }}$ bus. Using a perturbation matrix and network reduction [20], (9) is reduced as

$$
\left(\begin{array}{c}
\Delta \boldsymbol{V}_{1^{\epsilon}} \\
\Delta \boldsymbol{V}_{m^{\epsilon}}
\end{array}\right)=\boldsymbol{R}_{\mathrm{r}}\left(\begin{array}{c}
\Delta \boldsymbol{I}_{1}^{\epsilon} \\
\Delta \boldsymbol{I}_{m^{\epsilon}}
\end{array}\right) \text {, where } \boldsymbol{R}_{\mathrm{r}}=\left(\begin{array}{ll}
\boldsymbol{R}_{\mathrm{r}, 11} & \boldsymbol{R}_{\mathrm{r}, 12} \\
\boldsymbol{R}_{\mathrm{r}, 21} & \boldsymbol{R}_{\mathrm{r}, 22}
\end{array}\right)
$$

where $\boldsymbol{R}_{\mathrm{r}}$ is the resistance matrix for the reduced network. The sum of current change should be zero in the reduced network, which can be simplified to $\Delta \boldsymbol{I}_{1}{ }^{\epsilon}=-\Delta \boldsymbol{I}_{m^{\epsilon}}$ and it leads to $\Delta \boldsymbol{V}_{1^{\epsilon}}=\left(\boldsymbol{R}_{\mathrm{r}, 12}-\boldsymbol{R}_{\mathrm{r}, 11}\right) \Delta \boldsymbol{I}_{m^{\epsilon}}$. Then, using the relation of (10) and (11), the following is obtained

$$
\Delta \boldsymbol{V}_{L, 1^{\epsilon}}=\boldsymbol{K}_{\mathrm{r}} \Delta \boldsymbol{I}_{L, m^{\epsilon}}, \text { where } \Delta \boldsymbol{V}_{L, 1^{\epsilon}}=\left(\begin{array}{c}
\Delta V_{L, 1^{\epsilon}}^{+} \\
\Delta V_{L, 1^{\epsilon}}^{-}
\end{array}\right)
$$

where $\boldsymbol{K}_{\mathrm{r}}$ is calculated using (10), (11), and (18). Eq. (19) expresses the relation between the changes of the $\mathrm{V}$ bus line voltage and the currents absorbed into ESS. Assuming ESS charges with the same currents absorbed into the positive and negative sides $\left(\Delta I_{L, m^{\epsilon}}^{+}=\Delta I_{L, m^{\epsilon}}^{-}\right)$, we conclude 
Table 2. Simulation procedure

\begin{tabular}{c|l}
\hline Step A & $\begin{array}{l}\text { The grid's operating condition is given: For the V bus, voltage is given. For P buses, powers (powers generated by DRGSs and powers } \\
\text { consumed by loads) are given. }\end{array}$ \\
\hline Step B & By solving the power flow problem based on the operating condition, the information of voltages, currents, and powers is obtained. \\
\hline Step C & $\begin{array}{l}\text { Based on the information obtained in Step B, the optimization problem is solved and ESS power to be injected into the grid is obtained. } \\
\text { Then, the operating condition is updated. }\end{array}$ \\
\hline Step D & Go to Step A and perform the steps again. \\
\hline
\end{tabular}

Table 3. Cable length between buses in Fig. 1 (cable resistance $0.5 \Omega / \mathrm{km}$ )

\begin{tabular}{c|c|c|c|c|c|c|c|c|c|c|c|c|c|c|}
\hline Bus-to-bus & $1-6$ & $2-3$ & $3-4$ & $3-5$ & $5-11$ & $6-5$ & $6-7$ & $7-8$ & $7-10$ & $8-9$ & $9-12$ \\
\hline Length $(\mathrm{km})$ & 0.64 & 0.2 & 0.5 & 0.3 & 0.4 & 0.26 & 0.26 & 0.26 & 3.4 & 0.3 & 0.6 \\
\hline
\end{tabular}

$\Delta V_{L, 1^{\epsilon}}^{+}=\Delta V_{L, 1^{\epsilon}}^{-}$and (19) is simplified to $\Delta V_{L, 1^{\epsilon}}^{+}=K \Delta I_{L, m^{\epsilon}}^{+}$. Then, the reference for mode 2 is given as

$$
V_{L}^{*}=1.0-K \Delta I_{L, m^{\epsilon}}^{+}
$$

where $\Delta I_{L, m^{\epsilon}}^{+}=I_{L, m^{\epsilon}}^{+}-I_{L o, m^{\epsilon}}^{+}$and $I_{L, m^{\epsilon}}^{+}$should be less than the rated current of VSDGS. Here, we like to note that ESS is charged by reducing reference $V_{L}^{*}$, instead of increasing the $\mathrm{V}$ bus line voltage. For instance, suppose an operating condition in which $0.1 \mathrm{pu}$ and $0.05 \mathrm{pu}$ currents by VSDGS are injected into the V bus. If we like to make VSDGS generate additional $0.3 \mathrm{pu}$ and $0.3 \mathrm{pu}$ currents for ESS charging, the $\mathrm{V}$ bus line voltage should increase by $\Delta V_{L, 1^{\epsilon}}^{+}=K \times 0.3$. Instead, we can have the same result by reducing the reference.

\section{Simulation Results}

To verify the proposed method, a computer simulations was performed. The simulation procedure is outlined in Table 2. The grid configuration for the simulation was the same as the one shown in Fig. 1(a). For convenience, the superscript ' $\epsilon$ ' is ignored when indicating the bus numbers in this section. The base power and voltage were chosen as $100 \mathrm{~kW}$ and $750 \mathrm{~V}$. The ESS capacity was chosen as 250 $\mathrm{kWh}$. The cable resistance for the grid is listed in Table 3.

\subsection{Simulation result one}

For the simulation in this sub-section, the operating condition is given as listed in Table 4 .

Based on the operating condition, the results without and with ESS are given in Tables 5 and 6. In Table 5, the powers at bus 1 that VSDGS is connected, and at bus 2 that ESS is connected, are stated. The power generated from DRGSs is greater than that consumed by the loads. Therefore, without ESS, some of the generated power should be dissipated by a resistor equipped with DVSG. On the other hand, the power to be wasted can be minimized by applying ESS. In Table 6, the line voltages at the buses are listed. Without ESS, the positive and negative components of the line voltages are unbalanced. On the
Table 4. Initial operating condition for the DC grid

\begin{tabular}{|c|c|c|c|c|c|}
\hline \multicolumn{2}{|c|}{ Bus } & \multirow{2}{*}{$\begin{array}{c}\text { Voltage } \\
\text { (p.u) }\end{array}$} & \multicolumn{3}{|c|}{ Power $(\mathrm{kW})$} \\
\hline No. & Type & & Type & Consumed & Generated \\
\hline 1 & V bus & $1,0,-1$ & VSDGS - type $3(p, n)$ & - & - \\
\hline 2 & \multirow{11}{*}{$\mathrm{P}$ bus } & - & ESS - type $3(p, n)$ & - & - \\
\hline 3 & & - & & . & . \\
\hline 4 & & - & Load - type 4 (p-n) & 10.18 & $\cdot$ \\
\hline 5 & & - & Load - type $2(n)$ & 13.39 & 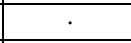 \\
\hline 6 & & - & Load-type $3(p, n)$ & $1.6,9.8$ & . \\
\hline 7 & & - & Load - type $3(p, n)$ & $2.49,9.92$ & . \\
\hline 8 & & - & Load - type $1(\mathrm{p})$ & 11.18 & . \\
\hline 9 & & - & Load - type 4 (p-n) & 15.26 & . \\
\hline 10 & & - & Load - type 4 (p-n) & 12.1 & . \\
\hline 11 & & - & PV - type $3(p, n)$ & $\cdot$ & 55,55 \\
\hline 12 & & - & Wind - type $4(p-n)$ & . & 25 \\
\hline
\end{tabular}

Table 5. Powers injected to bus 1 and 2 without and with ESS

\begin{tabular}{c|c|c|c|c}
\hline \multirow{2}{*}{ Bus No. } & \multicolumn{2}{|c|}{ Without ESS } & \multicolumn{2}{c}{ With ESS } \\
\cline { 2 - 5 } & Pos. & Neg. & Pos. & Neg. \\
\hline $\begin{array}{c}1 \\
\text { (VSDGS) }\end{array}$ & $\begin{array}{c}-31.79 \\
\text { (wasted) }\end{array}$ & $\begin{array}{c}-14.85 \\
\text { (wasted) }\end{array}$ & -0.08 & 3.85 \\
\hline $\begin{array}{c}2 \\
\text { ESS) }\end{array}$ & 0.0 & 0.0 & $\begin{array}{c}-32.14 \\
\text { (charged) }\end{array}$ & $\begin{array}{c}-18.65 \\
\text { (charged) }\end{array}$ \\
\hline
\end{tabular}

Table 6. Line voltages without and with ESS

\begin{tabular}{c|c|c|c|c}
\hline \multirow{2}{*}{ Bus No. } & \multicolumn{2}{|c|}{ Without ESS } & \multicolumn{2}{c}{ With ESS } \\
\cline { 2 - 5 } & Pos. & Neg. & Pos. & Neg. \\
\hline 1 & 1 & 1 & 1 & 1 \\
\hline 2 & 1.023 & 1.002 & 0.991 & 0.998 \\
\hline 3 & 1.023 & 1.002 & 0.996 & 0.998 \\
\hline 4 & 1.021 & 1.000 & 0.995 & 0.997 \\
\hline 5 & 1.023 & 1.003 & 1.004 & 0.999 \\
\hline 6 & 1.016 & 0.999 & 1.001 & 0.997 \\
\hline 7 & 1.013 & 0.998 & 0.999 & 0.996 \\
\hline 8 & 1.011 & 1.001 & 0.997 & 0.999 \\
\hline 9 & 1.012 & 1.001 & 0.997 & 0.999 \\
\hline 10 & 1.003 & 0.988 & 0.989 & 0.986 \\
\hline 11 & 1.034 & 1.014 & 1.014 & 1.011 \\
\hline 12 & 1.015 & 1.005 & 1.001 & 1.003 \\
\hline Avg. & 1.016 & 1.001 & 0.999 & 0.999 \\
\hline
\end{tabular}

other hand, by applying ESS, the line voltages become well balanced and closer to 1.0 pu than the case without ESS.

Fig. 4 shows power flow results in the test system with ESS. Unlike the powers and voltages stated in Tables 5 and 


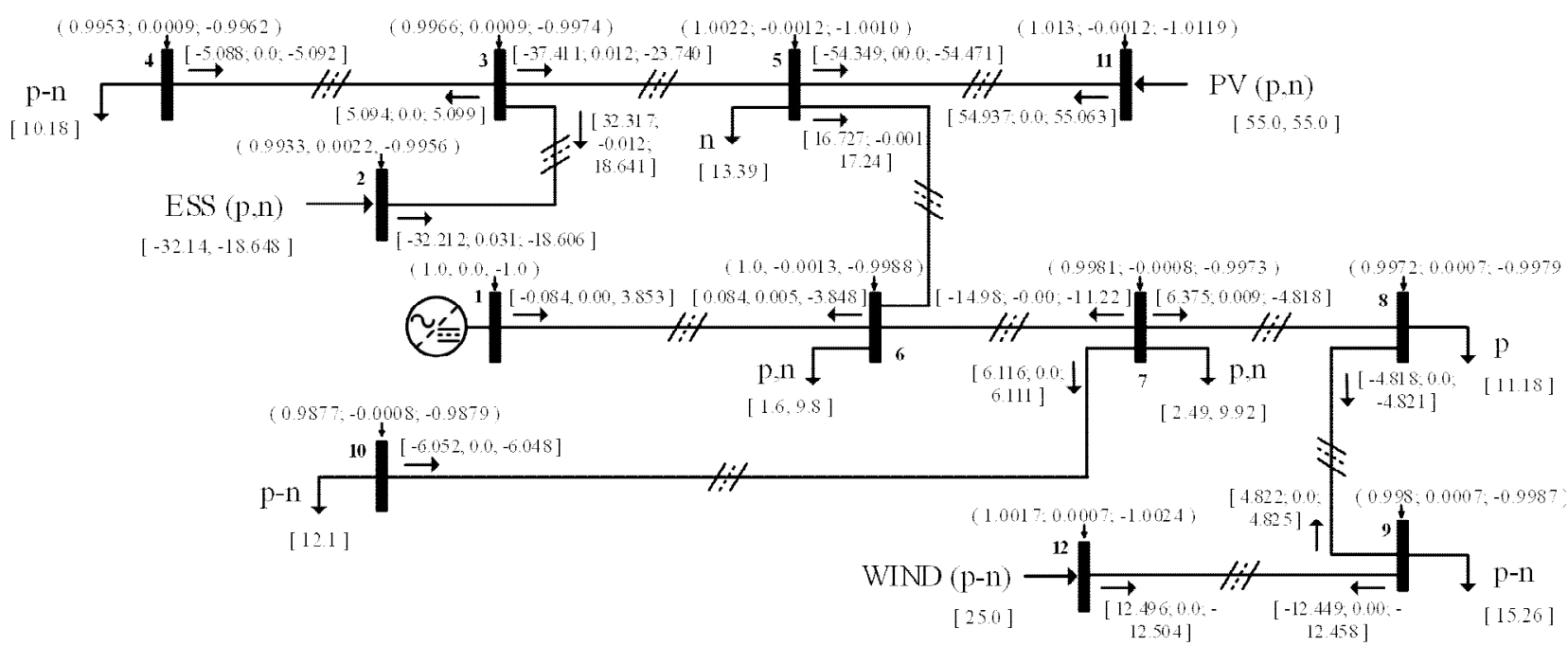

Fig. 4. Test system of bipolar DC micro-grid with ESS (power flow displayed)
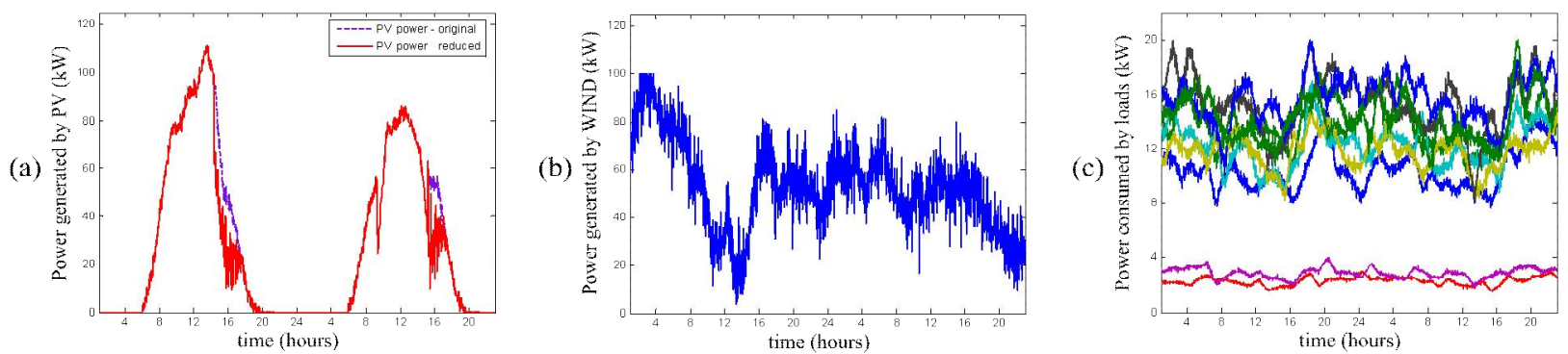

Fig. 5. (a) Power generated by PV; (b) power generated by wind; (c) power consumed by loads


Fig. 6. (a) Power generated by VSDGS; (b) ESS power; (c) ESS SoC with mode 1
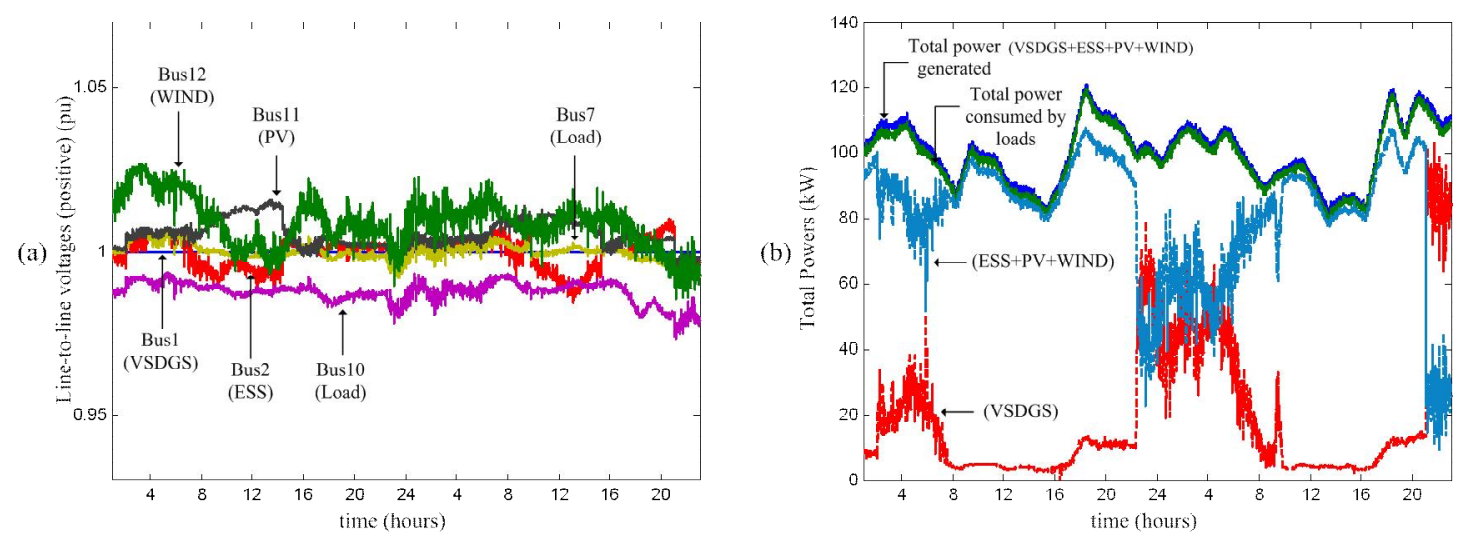

Fig. 7. (a) Positive line voltages of chosen buses; (b) Summed powers of generation sources and loads with mode 1 
6 , the figure shows the bus voltages and bus powers, where the bus powers are calculated based on the bus voltages and line currents. It is evident that, for instance, the bus powers from bus 1 and bus 6 are different on account of the line losses. Furthermore, the powers generated by the positive and negative sides of $\mathrm{PV}$ at bus 11 are equally 55 and $55 \mathrm{~kW}$, respectively. However, the bus powers flowing from Bus 11 are not equally distributed because of the different bus voltages. The powers flowing from the positive, neutral, and negative buses are 54.937, 0, and $55.063 \mathrm{~kW}$, respectively.

\subsection{Simulation result two}

The ESS operation is often restricted by SoC. To observe the effect by SoC, long-term simulations must be performed. Therefore, mode 1 was first used for the charging. The results are shown in Figs. 4, 5, and 6. Then, mode 2 was used for the charging. The results are shown in Figs. 7 and 8 .

Fig. 5(a) shows the power generated by PV, where the dashed line denotes the power to be fully obtained; the solid line represents the reduced power to avoid a power surplus. Fig. 5(b) shows the power generated by wind, and Fig. 5(c) depicts the power consumed by loads. Fig. 6(a) illustrates the power generated by VSDGS, and Fig. 6(b) shows the ESS power. Fig. 6(c) depicts ESS SoC. ESS charged between 8 to $14 \mathrm{~h}$. Therefore, VSDGS had to waste power generated by DRGSs. Between 16 to $22 \mathrm{~h}$, ESS discharged; thus, VSDGS generated much less power than that required by the loads. Between 22 and the next 8 $\mathrm{h}$, ESS SoC was at its lower limit so that VSDGS produced more power to meet the load demands. Fig. 7(a) shows the chosen bus positive line voltages. It is apparent that, when DRGSs produce power, the respective bus line voltages are higher than that of VSDGS. Fig. 7(b) shows the summed powers of generation sources and loads, where we see the total line loss remains small. When power from DRGSs is small and ESS cannot produce power by the low limit, VSDGS copes with the load demand alone.

Fig. 8(a) depicts the power generated by VSDGS, while Figs. 8(b) and (c) show the ESS power and ESS SoC, respectively. At approximately 3 to $4 \mathrm{~h}$ and the next 8 to 9 h, ESS is rapidly charged by power from VSDGS. Fig. 9(a) shows the chosen bus positive line voltages. It is evident that bus 2, to which ESS is connected, has lowest line voltage during the rapid charging of ESS. In Fig. 9(b), when ESS is charged between 3 and $4 \mathrm{~h}$, the total power generated is quite larger than the total power demand, which means that the power difference is wasted as a line loss. This is logical because, when ESS is charged, ESS works as a load; that is, the total demand is more than doubled.
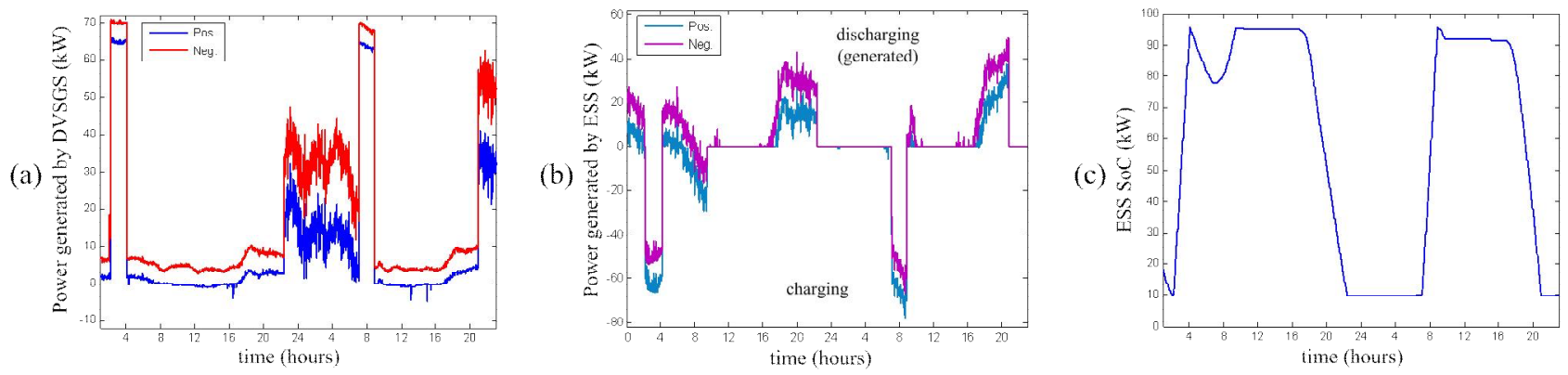

Fig. 8. (a) Power generated by VSDGS, (b) ESS power, (c) ESS SoC with mode 2


Fig. 9. (a) Positive line voltages of chosen buses, (b) Summed powers of generation sources and loads with mode 2 


\section{Conclusion}

In general, the power flow problem is considered a basic method for identifying the complete operating conditions in a power grid system. In a low-voltage DC grid system, line impedances are no longer negligible if the grid expands with long lines. Thus, the power flow problem is required for analysing the grid system. Therefore, this paper introduced and solved the power flow problem for the bipolar-type of DC micro-grid system, including unbalanced load conditions. In addition, this paper introduced a control strategy for ESS installed in a DC micro-grid, which employs the simple principle implying that power from VSDGS and power flowing into the DC micro-grid are minimized by minimizing the voltage differences between buses. Using computer simulations, the usefulness of the introduced methods was verified. Last, we like to remind the readers that the power flow study is concerned with steady state analysis, and thus the simulation results do not produce any of transient dynamics.

\section{References}

[1] S. Backhaus, G. W. Swift, S. Chatzivasileiadis, W. Tschudi, S. Glover, M. Starke, J. Wang, M. Yue, and D. Hammerstrom "DC Microgrids Scoping Study: Estimate of Technical and Economic Benefits (March 2015)," US Department of Energy, 2015. [Online]. Available: http://energy.gov/oe/downloads/dcmicrogrids-scoping-study-estimate-technical-andeconomic-benefits-march-2015

[2] P. Savage, R. R. Nordhaus, and S. P. Jamieson, "DC Microgrids: Benefits and Barriers," From Silos to Systems: Issues in Clean Energy and Climate Change, pp. 51-66, 2010.

[3] A.T. Ghareeb, A.A. Mohamed, and O.A. Mohammed, "DC microgrids and distribution systems: An overview," Power and Energy Society General Meeting (PES), IEEE, 2013, pp. 1-5.

[4] K. Hirose, T. Tanaka, T. Babasaki, S. Person, O. Foucault, B. J. Sonnenberg, and M. Szpek, "Grounding concept considerations and recommendations for 400VDC distribution system," Telecommunications Energy Conf. (INTELEC), IEEE, 2011, pp. 1-8.

[5] M. Noritake, K. Yuasa, T. Takeda, H. Hoshi, and K. Hirose, "Demonstrative research on DC microgrids for office buildings," Telecommunications Energy Conf. (INTELEC), IEEE, 2014, pp. 1-5.

[6] P. Nuutinen, T. Kaipia, P. Peltoniemi, A. Lana, A. Pinomaa, P. Salonen, J. Partanen, J. Lohjala, and M. Matikainen, "Experiences from use of an LVDC system in public electricity distribution," Electricity Distribution (CIRED), 2013, pp. 1-4.

[7] H. Kakigano, Y. Miura, T. Ise, and R. Uchida, "DC micro-grid for super high quality distribution - system configuration and control of distributed generations and energy storage devices," Power Electronics Specialists Conf. (PESC), IEEE, 2006, pp. 1-7.

[8] S. Whaite, B. Grainger, and A. Kwasinski, "Power quality in DC power distribution systems and microgrids," Energies, vol. 8, no. 5, pp. 4378-4399, 2015.

[9] D. Palit and A. Chaurey, "Off-grid rural electrification experiences from South Asia: Status and best practices," Energy for Sustainable Development, vol. 15, no. 13, pp. 266-276, Sept. 2011.

[10] T. Mishimaa, I. Taniguchib, H. Tamakic, Y. Kitagawad, K. Yutanie, and K. Suekanef, "A verify-cation of high-efficiency DC micro-grid power systems with high-performance power converters and energy management strategy," Procedia Technology, vol. 18, pp. 47-52, Sept. 2014.

[11] C. Abbey, D. Cornforth, N. Hatziargyriou, K. Hirose, A. Kwasinski, E, Kyriakides, G. Platt, L. Reyes, S. Suryanarayanan, "Powering through the storm: microgrids operation for more efficient disaster recovery," IEEE Power and Energy Magazine, vol. 12, no. 3, pp. 67-76, 2014

[12] J. J. Justoa, F. Mwasilua, J. Leeb, J.-W. Jung, “ACmicrogrids versus DC-microgrids with distributed energy resources: A review," Renewable and Sustainable Energy Reviews, vol. 24, pp. 387-405, Aug. 2013.

[13] H. Kakigano, Y. Miura, and T. Ise, "Low-voltage bipolar-type DC micro-grid for super high quality distribution," IEEE Trans. on Power Electrons., vol. 25, no. 12, pp. 3066-3075, Dec. 2010.

[14] B. Liu, F. Zhuo, Y. Zhu, and H. Yi, "System operation and energy management of a renewable energy-based DC micro-grid for high penetration depth application," IEEE Trans. on Smart Grid, vol. 6, no. 3, pp. 11471155, May 2015.

[15] N. Eghtedarpour and E. Farjah, "Distributed charge/ discharge control of energy storates in a renewableenergy-based DC micro-grid," IET Renewable Power Gen., vol. 8, no. 1, pp. 45-57, Jan. 2014.

[16] S.-y. Lu, L. Wang, T.-M. Lo, and A. V. Prokhorov, "Integration of wind power and wave power generation systems using a DC microgrid," IEEE Trans. on Ind. Applicat., vol. 51, no. 4, July/Aug. 2015.

[17] C. L DeMarco, C. A. Baone, Y. Han, and B. Lesieutre, "Primary and secondary control for high penetration renewables," PSERC Publication, May 2012. [Online]. Available: http://pserc.wisc.edu/recent_publications.aspx

[18] A. R. Bergen and V. Vittal, 'Power systems analysis' (Prentice-Hall Press., 1986, 2nd edn. 2000)

[19] M. Grant and S. Boyd: CVX - MATLAB software for disciplined convex programming, version 2.0 beta. http://cvxr.com/cvx, Sept. 2013.

[20] D.-E. Kim, and M. A. El-Sharkawi "Dynamic equivalent model of wind power plant using an aggregation technique," IEEE Trans. on Energy Conv., vol. 30, no. 4, pp. 1639-1649, Dec. 2015. 
Dong-Eok Kim received the B.S. and M.S. degrees in electrical engineering from Yeungnam University, Gyeongbuk, Korea, in 2005 and 2008, respectively, and the $\mathrm{Ph} . \mathrm{D}$. degree in electrical engineering from the University of Washington, Seattle, WA, USA, in 2014. Currently, he is a Senior Researcher at KEPCO Research Institute, Daejeon, Korea. His research interests include power system modeling, dynamics, and fault analysis, as well as control of power systems. 